\title{
High-risk HPVs, microbiota and epithelial carcinogenesis: state of the art and research contribution of in vitro 3D models
}

\author{
Diletta Francesca Squarzanti ${ }^{1,2, \#, ~ R i t a ~ S o r r e n t i n o, " \#, ~ B a r b a r a ~ A z z i m o n t i ~}{ }^{1,2,3}$ \\ 'Laboratory of applied Microbiology, Center for Translational Research on Allergic and Autoimmune Diseases, Department of \\ Health Sciences, Medical School, Università del Piemonte Orientale, Novara 28100, Italy. \\ ${ }^{2}$ Department of Health Sciences, Medical School, Università del Piemonte Orientale, Novara 28100, Italy. \\ ${ }^{3}$ Consorzio Interuniversitario Nazionale per la Scienza e Tecnologia dei Materiali, Firenze 50121, Italy (Local Unit of Università del \\ Piemonte Orientale, Novara 28100, Italy). \\ \#Authors contributed equally.
}

Correspondence to: Barbara Azzimonti, Center for Translational Research on Allergic and Autoimmune Diseases, Department of Health Sciences, Medical School, Università del Piemonte Orientale, Corso Trieste 15/A, Novara 28100, Italy.

E-mail: barbara.azzimonti@med.uniupo.it

How to cite this article: Squarzanti DF, Sorrentino R, Azzimonti B. High-risk HPVs, microbiota and epithelial carcinogenesis: state of the art and research contribution of in vitro 3D models. J Cancer Metastasis Treat 2019;5:73.

http://dx.doi.org/10.20517/2394-4722.2019.19

Received: 15 Jul 2019 First Decision: 5 Sep 2019 Revised: 24 Oct 2019 Accepted: 24 Oct 2019 Published: 8 Nov 2019

Science Editor: Ciro Isidoro Copy Editor: Jing-Wen Zhang Production Editor: Tian Zhang

\begin{abstract}
Persistent high-risk human papillomavirus (HR-HPV) infection is associated with anogenital and head \& neck squamous epithelial (HNSCC) tumors, which altogether cause about 550,000 new cases every year. Several evidences suggest that the microbiota could have a role on the inflammatory, epithelial mesenchymal transition and tumorigenesis processes promoted by HR-HPV infection, yet the mechanisms involved remain to be clarified. In this review we report the state of the art on this topic and on the most promising in vitro developed models for studying the host-pathogen interactions. Using MEDLINE, several terms were searched and combined to select the most pertinent papers. The investigation was limited to the international indexed articles published in PubMed in the last 10 years. This review reports the latest knowledge in the field of the microbial-associated anogenital tumors and HNSCC. In addition, we also discuss the in vitro epithelial culture systems that reproduce the pathophysiological features of the tumoral microenvironment and the in vivo response to microbial agents, thus representing a useful tool for analyzing at cellular and molecular levels the role played by infective agents in tumorigenesis.
\end{abstract}

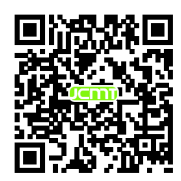


Keywords: High risk human papillomavirus, microbiota, Fusobacterium nucleatum, epithelial-mesenchymal transition, anogenital cancer, head \& neck squamous cell carcinoma, colorectal cancer, transepithelial electrical resistance

\section{INTRODUCTION}

As referred in the last human papillomavirus (HPV) cancer report of the HPV Information Centre ${ }^{[1]}$, unremitting HPV infection, mainly promoted by high-risk HPV (HR-HPV) 16 and 18 E6 and E7 oncoproteins, is the main microbial etiological factor till now recognized that is necessary, yet not sufficient, for the development of anogenital and of head \& neck cancers (namely of the tonsil, tongue and oropharynx).

Despite the progresses in vaccination and screening prevention led to the reduction of cases number ${ }^{[2,3]}$, these tumors are still diffuse among the general population, with the highest mortality rate in countries with a low socio-economic level ${ }^{[3]}$.

Cervical cancer is the 3rd most frequent female tumor in the world (2nd in the age range 15-44) with about 600,000 new cases/year and a 50\% mortality rate $e^{[4,5]}$.

In the last three decades oropharyngeal tumors, which include some 450,000 new cases/year (mostly affecting older tobacco and alcohol users worldwide), have increased also in young people, due to sexual habits changes predisposing to HR-HPV infection ${ }^{[6]}$.

It has been reported that oncogenic HPV-related oropharyngeal tumors have a better prognosis than those HPV negative ${ }^{[7,8]}$. On the other hand, it is known that HR-HPV-mediated cervical oncogenesis generally develops after a long latency (about 10-30 years from infection). Thus, it remains a conundrum the fact that tumor latency and prognosis differ in the two populations of patients, regardless of the treatment and of the fact that both types of cancer share a common infective cause ${ }^{[0]}$. Seemingly, HR-HPV infection is not per se enough to cause these cancers, though viruses are classified as Class 1 carcinogens by the International Agency for Research on Cancer ${ }^{[4]}$. Recent evidences suggest that the microbiota of the cervical and oropharyngeal niches (that includes bacteria, protozoa, viruses and fungi, although sometimes the term specifically refers only to bacterial communities) can have both a harmful and a protective role. Further, the microbiota can influence persistent HPV-mediated inflammation, epithelial mesenchymal transition (EMT) and tumorigenesis, although the mechanisms involved are still largely unknown and controversial ${ }^{[10]}$. In fact, just as a balanced bacteriota may be constituted by both pathogens and commensals, the human virota can include, among the others, both low risk and HR-HPVs, without inducing morbidity for its host if the immune surveillance and the whole microbiota itself are able to control its load and persistence ${ }^{[1]]}$.

Therefore, an open question is if commensal HPV types, belonging to the human virota, play a protective role against the oncogenic ones or not ${ }^{[12-15]}$. What is sure is that cancer often arises from a metabolic imbalance. Given this assumption, the metabolic profile of the microenvironment, in which host cells directly cooperate with viruses and bacteria, could reveal a snapshot of their functional and complex interactions that are at the basis of HPV infection, persistence susceptibility, inflammation, EMT, uncontrolled cell proliferation and cancer progression.

To learn more about it, researchers have developed several in vitro co-culture systems that could mimic the physiological appearance of the tumoral microenvironment ${ }^{[16]}$. 
On this basis, the purpose of this study was to show the state of the art on epithelial carcinogenesis and on the most suitable in vitro 3D models till now developed for the study of host-pathogens interactions.

By using MEDLINE, terms such as "microbiota", "virota", "virus", "epitheliotropic virus", "HPV", "bacteriota”, "bacteria”, "Fusobacterium nucleatum", "Porphyromonas gingivalis", "EMT”, "eukaryotic cells", "keratinocytes", "epithelial cells" "epithelial infection", "epithelial cancer", "anogenital cancer", "head and neck squamous cell carcinoma (HNSCC)", "epithelial models", “2D”, "3D”, “3D epithelial cultures", "rafts", "spheroids", "reconstructed tissues", both in single and mutually combined, were searched in titles and abstracts in order to find PubMed indexed most pertinent articles; only original research articles published in peer review journal were considered. Papers including EBV or other epitheliotropic viruses other than HPV were excluded.

The literature search was limited to the most recent scientific publications (10 years); six thousand and five hundred nine appropriate abstracts and full papers were carefully read and reviewed; those relevant (216) were reconsidered based on the above described inclusion and exclusion criteria and then 70 were reported in detail and included in the text.

Overall, our findings highlight the most recent advances in the field of host-pathogens interactions. We focused on the in vitro 3D modeling for the study of the microbial induced epithelial carcinogenesis and emphasized its ability in mimicking at best the role of bacteria and viruses within an in vivo microenvironment.

\section{MICROBIOTA, IMMUNE RESPONSE AND EPITHELIAL CANCER}

Several studies, among which that conducted by Kyrgiou et al. ${ }^{[10]}$, underlined that vaginal microbiota may drastically influence host innate immune response, infection susceptibility and cervical cancer development, but without proving causality and thus making necessary further longitudinal studies. In this direction, Ilhan et al ${ }^{[17]}$, through a triple approach based on liquid chromatography mass spectrometry, $16 \mathrm{~S}$ rRNA gene sequencing and immunoassay, evaluated and integrated the metabolic signature of the vaginal microbiota and the inflammatory status changes of 78 women, including HPV-negative/positive controls, low-/high-grade cervical-dysplasia or -cancer affected females. These authors found that unique cervicovaginal metabolites and microbes allow to discriminate clearly the affected patients from healthy subjects, thus providing novel cancer hallmarks. For example, the lipidic three-hydroxy-butyrate, eicosenoate, and oleate/vaccenate are distinctive of cancer patients, while sphingolipids, plasmalogens and linoleate positively correlate with cervicovaginal inflammation.

Regarding this niche, the absence of dysbiosis and a dominance of peculiar gram-positive bacteria belonging to the Lactobacillus genus may inhibit several pathogens, preserve aminoacidic and nucleotide metabolisms and avoid the inflammatory state. Conversely, Fusobacterium spp., F. nucleatum in primis, promotes cell survival, proliferation, dysregulated carcinogenesis and cervical cancer through the induction of the WNT/ Beta catenin signaling pathway, as emerged by research in colorectal cancer (CRC $)^{[12,18,19]}$.

Since it has been demonstrated a significant correlation between bacteria belonging to the Fusobacteria genus, mainly Sneathia sanguinegens, and high-grade squamous intraepithelial lesions, they could be hired as microbiological markers of clinically significant cervical diseases ${ }^{[10]}$.

This evidence has also been observed in another study by Mitra et $a l^{[12]}$, who found that a relative abundance of Fusobacterium spp. associates with higher levels of cytokines such as IL-4 and TGF-1 $\beta$, which have been shown to generate local immunosuppression, HPV immune evasion and cancer development. 
Moreover F. nucleatum inversely correlates with $\mathrm{CD}^{+}-\mathrm{T}$ cell counts, whose aberrant signaling and function is typical in cervical carcinogenesis.

Al-Hebshi et al ${ }^{[15]}$ have reported an abundance of the periodontal pathogen F. nucleatum, selected by the tumor environment, followed to a lesser extent by Campylobacter, Parvimonas and Prevotella spp. in oral squamous cell carcinoma, while the Streptococci are frequently associated with a healthy status.

Although the results obtained from clinical studies vary in the oral cancer associated microbiota composition, partially due to differences in the methodological approaches, the functional data consistently show an enrichment of a virulent, chronic inflammatory bacteriota that, as a passenger within the tumor environment, contributes to the worsening of the tumoral disease.

\section{HIGH-RISK HPVS, MICROBIOTA, EPITHELIAL MESENCHYMAL TRANSITION AND CANCER PROGESSION}

As known, EMT associates with cell migration, invasion, resistance to chemotherapy and anoikis. During this process, several signaling pathways, such as those involving TGF- $\beta$, Wnt, Hedgehog, $\beta$-catenin, Notch, Nanog, STAT3 and ERK, are activated in the cell leading to a pro-metastatic behavior ${ }^{[20,21]}$. In this context, EMT can be also regulated by HR-HPVs ${ }^{[22,23]}$.

Despite anogenital and oropharyngeal squamous cell carcinomas (OPSCC) promoted by these viruses have a more favorable prognosis respect to HPV negative ones, EMT usually associates with an aggressive tumoral behavior, making HPV role an open issue. In a study on a cohort of 296 OPSCC-patients, EMT was confirmed as an adverse marker of evolution of HPV related carcinomas ${ }^{[24]}$.

In another research, the expression of five EMT markers was assessed in normal and tumoral cervical tissues, revealing that $\mathrm{E}$-cadherin and $\beta$-catenin expression decreases gradually, while that of $\mathrm{N}$-cadherin, vimentin and fibronectin increases during malignant progression ${ }^{[25]}$. Moreover, Su et al ${ }^{[26]}$ studied the role of DNA methylation of the ten-eleven translocation methyl-cytosine dioxygenase 1 (TET1), which oxidizes 5-methylcytosine to 5-hydroxymethylcytosine within cervical lesions. At a precancerous level, TET1 inhibits EMT, interacting with chromatin modifiers and downregulating mesenchymal genes such as $Z E B 1$ and $V I M^{[26]}$. In vitro culture of cervical cancer cells revealed that $\mathrm{p} 68$, an ATP-dependent RNA helicase, induces EMT through the transcriptional activation of the TGF- $\beta 1$ pathway, inducing cancer $\operatorname{progression}^{[27]}$.

Finally, Pang et al. ${ }^{[28]}$ reported that HPV18 E6 regulates YB-1 mRNA expression by promoting EMT and cervical lesion progression through the enhanced Snail expression.

Moreover, both HPV and EMT upregulate programmed cell death ligand-1 (PD-L1) expression, an immune checkpoint and therapeutic target in OPSCC, whose efficacy is currently under investigation ${ }^{[29,30]}$. Precisely, PD-L1 is overexpressed in about half of OPSCC that seem to have a better response to anti-PD-1 therapy ${ }^{[31]}$.

Regarding the involvement of bacterial microbiota in EMT, F. nucleatum has been frequently found in stools and bioptic specimens of CRC affected patients, and more recently also of OPSCC patients. It has been suggested that this bacterium triggers EMT to malignant transformation by interacting with E-cadherin, as suggested by in vitro experiments on NCM460 cells ${ }^{[32,33]}$. In a cohort of stage III/IV CRC affected patients, Yan et al. ${ }^{[34]}$ found a significant correlation between the presence of F. nucleatum and tumor progression also in relation to chemotherapy efficacy, suggesting its role as a biomarker for a 
therapeutic approach improvement. Panebianco et al. ${ }^{[35]}$ also confirmed this link. Moreover, the same authors underlined that not only gut bacteria, but also the intratumor ones, may influence patients' response toward chemo- and immuno-therapeutic drugs and vice versa ${ }^{[36]}$.

More recently, it has emerged that EMT can also be promoted by Porphyromonas gingivalis, an anaerobe frequently found in chronic periodontitis. In fact, it has been observed that this bacterium causes an early EMT of chronically infected human oral epithelial cells (OECs) that, through PI3K/Akt activation, ultimately lead to the loss of E-cadherin and to the accumulation of $\beta$-catenin, together with an increased expression of Zeb1, vimentin, MMP-2, -7, and -9. Moreover, upon stimulation with this bacterium, OECs migratory capacity increases proportionally ${ }^{[37-39]}$.

Both F. nucleatum and P. gingivalis determine an enhanced transcription of mesenchymal markers, an increase of TGF- $\beta 1$, TNF- $\alpha$ and EGF and a downregulation of those associated to epithelial layer integrity, as evidenced by transepithelial electrical resistance measures ${ }^{[40]}$.

Recently, a new tumor metastasis model has been proposed. Till now it was supposed that epitheliallike cancer cells acquire mesenchymal features in order to produce metastasis by exploiting the vascular system, and then switch back to an epithelial phenotype to establish the new tumor. The existence of a stable population of hybrid epithelial/mesenchymal cells, possessing epithelial and mesenchymal characteristics with both tumorigenic and metastatic properties, has been hypothesized ${ }^{[41,42]}$. Moreover, in predisposed individuals, with defects in the innate immunity response or with a specific epigenetic background, bacterial or viral infections could lead to EMT, causing a chronic inflammatory state, through the activation NF-kB and MAPK ${ }^{[43]}$. Considering this and the emerging role of the microbiota on EMT and cancer progression, more rational and shared laboratory models should be used to better investigate the microbiota composition ${ }^{[44]}$ and its relationship with the human host.

\section{IN VITRO EPITHELIAL MODELS FOR STUDYING THE HOST-PATHOGENS INTERACTIONS}

The first studies focused on host-pathogens connections started in the 1970 s with Todaro $^{[45]}$ and TaylorRobinson ${ }^{[46]}$, who described the interactions between oncogenic viruses and human cells, and mycoplasma pneumonia and ciliated tracheal epithelium, respectively. Since then, in vitro models acquired popularity in the microbiology field, due to their reproducibility, higher versatility and high-throughput data acquisition respect to in vivo models ${ }^{[47]}[\text { Table } 1]^{[5,16,38,45-62]}$. Undeniably, due to the improvement in imaging and screening techniques and to the in vitro models features, nowadays it is possible to directly analyse characteristics such as: specific surface-adhesion processes (i.e., biofilm formation), spreading capabilities, immune system escapes aptitudes, migration in $3 \mathrm{D}$ matrices, and host's cell-type-specific interactions in general.

The development of functional co-culture systems mostly depends on their ability to allow the physiological growth of cellular components in optimized culture media inside trans-well devices or microfluidic chambers. In this regard, Di Giulio et al. ${ }^{[50]}$ developed a model in which Streptococcus mitis was grown with human gingival fibroblasts in saliva sterilized stocks enriched with $1 \%$ sucrose as culture medium. With this system, the authors demonstrated that $S$. mitis can penetrate within fibroblasts, via a FAK-integrin $\beta 1$ vinculin-actin mediated process, which is modulated by the saliva and the microenvironment themselves.

Sterilized artificial saliva has also been used in more complex models such as the multi-culture trans-well system developed by Millhouse et al. ${ }^{[51]}$, in which a complex multispecies biofilm, composed by S. mitis, F. nucleatum, P. gingivalis and Aggregatibacter actinomycetemcomitans, was grown on a glass slide and cocultivated in a trans-well together with immortalized oral keratinocytes (OKF6-TERT2). With this system, these authors validated the efficacy of commercially available oral hygiene products. 
Table 1. Epithelial study models' overview (images created with BioRender.com)

\begin{tabular}{|c|c|c|c|c|}
\hline Epithelial study models & Since & Pros & Cons & Ref. \\
\hline P & $1920 \mathrm{~s}$ & $\begin{array}{l}\text { In vitro reproducibility; versatility; high- } \\
\text { throughput data acquisition }\end{array}$ & $\begin{array}{l}\text { Inability to reproduce the in } \\
\text { vivo context; poor biological } \\
\text { complexity; lack of the 3D; } \\
\text { poor data reliability; no cell-cell } \\
\text { interaction }\end{array}$ & $\begin{array}{l}\text { Todaro et al. }{ }^{[45]} \\
\text { Bergmann et al. }{ }^{[47]}\end{array}$ \\
\hline In vivo models & 1980s & $\begin{array}{l}\text { Suitability for: (1) new therapies and } \\
\text { preventative treatments testing; ( } 2 \text { ) } \\
\text { biological processes understanding }\end{array}$ & $\begin{array}{l}\text { Inability to recapitulate the } \\
\text { human tissue context; time- } \\
\text { consuming procedures; high } \\
\text { costs; requirement of specific } \\
\text { manual skills; ethical issues }\end{array}$ & $\begin{array}{l}\text { Taylor-Robinson et al. }{ }^{[46]} \\
\text { Pasupuleti et al. }{ }^{[48]}\end{array}$ \\
\hline 3D spheroids & 1980s-90s & $\begin{array}{l}\text { Fully humanized models; no animal } \\
\text { sacrifice required }\end{array}$ & $\begin{array}{l}\text { No tissue organization; } \\
\text { unsatisfactory biological } \\
\text { complexity; insufficient } \\
\text { data reliability; lack of cell- } \\
\text { microenvironment interactions }\end{array}$ & $\begin{array}{l}\text { Melissaridou et al. }{ }^{[16]} \\
\text { Bergmann et al. }{ }^{[47]} \\
\text { Sawant et al. }{ }^{[61]}\end{array}$ \\
\hline 3D organotypic cultures & 1990s-2000 & $\begin{array}{l}\text { Fully humanized models; no animal } \\
\text { sacrifice required; capacity to mimic the } \\
\text { in vivo context: recapitulation of epithelial } \\
\text { strata, cytokeratin differentiation, EMT } \\
\text { markers expression and carcinogenic } \\
\text { process; possibility of implementation } \\
\text { with the vascular counterpart, } \\
\text { macrophages and T-cells; permissiveness } \\
\text { to the physiological growth of cellular } \\
\text { components; appreciable biological } \\
\text { complexity; suitability for the study of } \\
\text { pathogen-induced cells modification and } \\
\text { host tissue-microbial agents interactions; } \\
\text { more reliability and cheaper to antiviral } \\
\text { drugs properties study than xenografts; } \\
\text { reliability of the data }\end{array}$ & $\begin{array}{l}\text { Model management } \\
\text { complexity; inability to mimic } \\
\text { long-term conditions }\end{array}$ & $\begin{array}{l}\text { Squarzanti et al. }{ }^{[5]} \\
\text { Sztukowska et al. }{ }^{[38]} \\
\text { Riedl et al. }{ }^{[49]} \\
\text { Di Giulio et al. }{ }^{[50]} \\
\text { Millhouse et al. } .^{[51]} \\
\text { Bradbury et al. }{ }^{[56]} \\
\text { Genovese et } a l^{[53]} \\
\text { Banerjee et al. }{ }^{[54]} \\
\text { Chow et al. }{ }^{[55]} \\
\text { Spurgeon et al. }{ }^{[52]} \\
\text { Hogervorst et al. }{ }^{[57]} \\
\text { Fullar et al. } \\
\text { de Carvalho Dias et al. }{ }^{[59]} \\
\text { Zhang et al. }{ }^{[60]} \\
\text { Dabija-Wolter et al. }{ }^{[62]}\end{array}$ \\
\hline
\end{tabular}

EMT: epithelial mesenchymal transition

Despite these findings and the evidences on the cytoskeletal modifications, the impaired proliferation rate and the gene expression changes occurring in cells infected by bacterial or viral pathogens ${ }^{[50,63-66]}$, some limitations arise when more complex evaluations are required. According to the literature, the microenvironment highly modifies cells behaviour; therefore, the lack of the " $3 \mathrm{D}$ " in many in vitro models reduces the transferability potential of the in vitro data ${ }^{[52]}$. This is particularly true for epitheliotropic viruses, such as HPVs, for which the intracellular expression of viral proteins is conditioned by the epithelial renewal/stratification process that cannot be detected within $2 \mathrm{D}$ cultures.

In fact, most 3D models enable not only viral life cycle, but also virus induced cellular modifications. They are generally constituted by HPV infected keratinocytes, cultivated onto fibroblast repopulated matrices and let to stratify at the air-liquid interface in specific culture media. They can be easily analysed by western blot, histology, high throughput sequencing and protein-protein interaction assays.

These models made possible to identify novel pro-oncogenic co-factors, such as $130^{[53]}$ or $53 \mathrm{BP} 1^{[5]}$, important for investigating novel antiviral targets.

3D RAFT (real architecture for 3D tissue) cultures also allowed to elucidate the effect of HPV chronic infection onto eukaryotic cells mitosis; as showed by Banerjee et al. ${ }^{[5]}$, HPV DNA amplification occurs during host's cell G2 phase, despite DNA eukaryotic amplification normally occurs in the S phase; in particular, they elucidated the key role of E7 in forcing host cells permanence in the G2 phase via cyclin B1 cytoplasmic accumulation. 
Additionally, RAFT cultures are a more reliable and cheaper solution to study antiviral drugs respect to xenografts in SCID mice, since they are more suitable to be analysed by in situ hybridization and RT-qPCR techniques $^{[55]}$.

Moreover, they can allow the study of host-virus interactions in absence of other cell types or tissues that could hide fundamental pathways promoted by the pathogen, especially when the studies require miRNA or EMT analysis.

As previously described, HR-HPVs E6 and E7 proteins interact and inhibit host p53 and pRb oncosuppressors respectively. Recently, the idea that E6 and E7 can also bind host miRNA or miRNA regulators has been evaluated ${ }^{[67]}$. Indeed, despite HPV inability to encode for miRNAs, HPV mediated indirect regulation of both onco-suppressive or tumorigenic miRNAs may offer the opportunity to identify novel therapeutic targets.

\section{RAFT CULTRES, DRUG RESISTANCE AND EMT RESEARCH}

Regarding drug resistance and EMT markers expression analysis, RAFT cultures can overcome both 2D and spheroid cultures limitations. Melissaridou et al ${ }^{[16]}$, working with 5 HNSCC-derived cell lines grown in both $2 \mathrm{D}$ and spheroids assays, observed that, while $2 \mathrm{D}$ eukaryotic cells and spheroid conformation changes the receptor topography and gene expression, and erases the drug resistance pathways that occur in vivo, $3 \mathrm{D}$ cultures can better mimic what happens during in vivo therapies and allow to study EMT expression markers such as CDH1, NANOG, SOX and EGFR.

However, when epithelial models are necessarily to be used, spheroids utility is limited, because of the less reliability of the data they produce respect to those obtained with the organotypic models. In fact, although they are both made up of epithelial cells, spheroids morphology and structure are too much simplified and do not reproduce the mucosal epithelium in such a representative way as the $3 \mathrm{D}$ epithelial RAFTs do. Therefore, compared to 3D RAFTs, the spheroids are less performant for studies on the specific interactions that occur in a well-defined in vivo environment.

On the other hand, RAFT cultures can be built with both isolated healthy or tumour/cancer associated keratinocytes and fibroblasts, able to better elucidate the interactions between stromal and epithelial compartments that are at the basis of the initial steps of the carcinogenetic process ${ }^{[56]}$. Moreover, they recapitulate all the epithelial layers, the physiological cytokeratin differentiation and the carcinogenic process.

By using in vitro reconstructed epithelial systems, Hogervorst et al. ${ }^{[57]}$ highlighted the importance of the papillary-reticular fibroblasts switch. In fact, when epithelial cultures are grown in presence of switched fibroblasts, the expression of CAF associated markers ( $\alpha$-SMA and vimentin) and EMT markers (SNAIL2, $\mathrm{N}$-cadherin and ZEB1) increase, thus suggesting that the fibroblasts switch could be considered an indicator of squamous cell carcinoma (SCC) progression. Similarly, Fullar et al.$^{[58]}$ proved that HPV16 induces the modification of connective matrix that, by the action of epithelial Matrix Metalloproteinase MMP-7 and fibroblast-produced MMP-2, loses collagen and fibronectin in favour of laminin, thus facilitating EMT and carcinogenic events. Comparable effects on MMP super-families were also observed in other HR-HPV positive models ${ }^{[68]}$.

In agreement with the above, the last trend is to reproduce tissue models with the whole organ complexity ${ }^{[69]}$; in fact, the models have been implemented with the vascular component, with macrophages and T-cells, thus allowing to further improve the knowledge in the microbiology field ${ }^{[59-61]}$. 


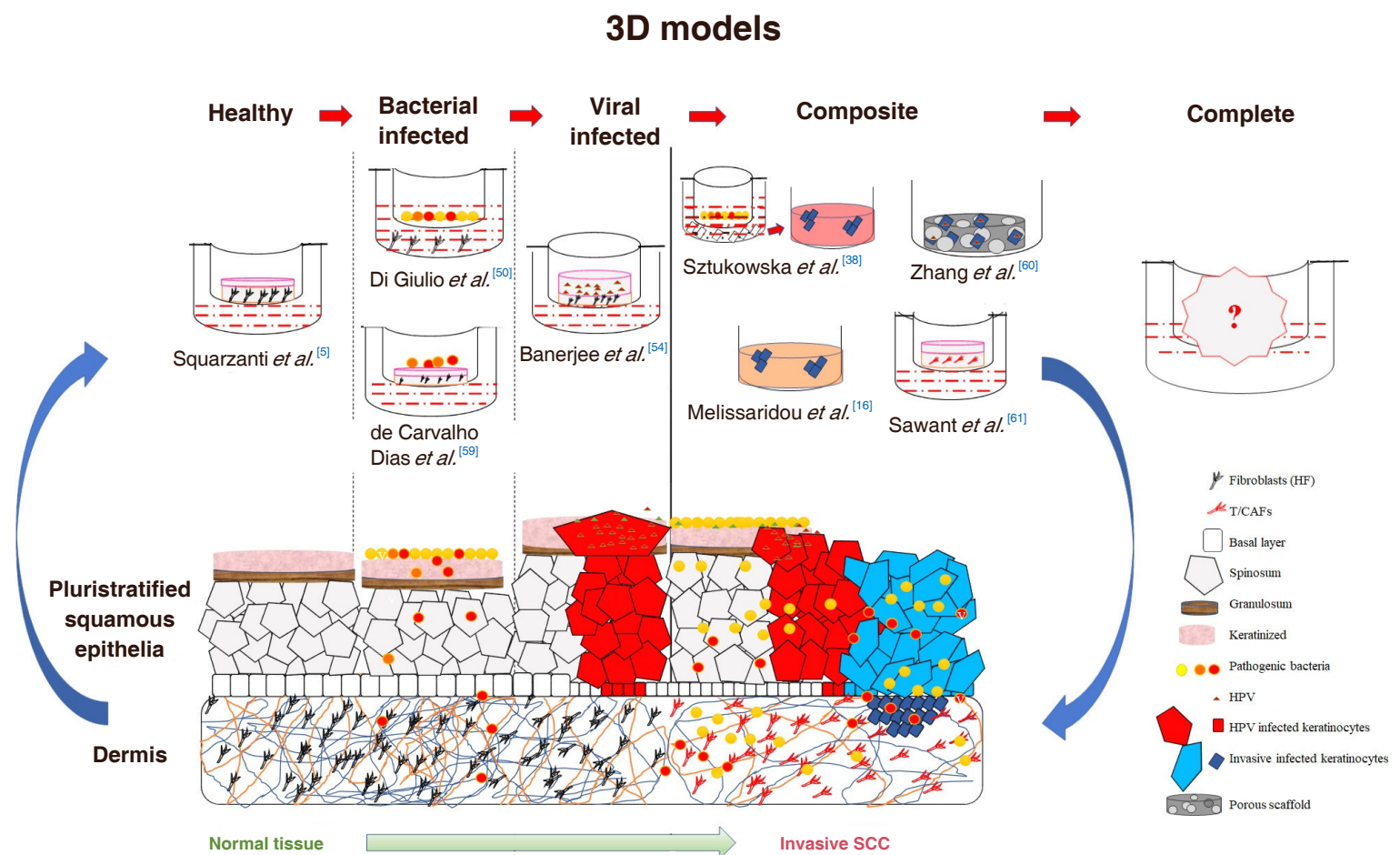

Figure 1. Schematic representation of SCC progression achievable by using in vitro 3D infected models. SCC: squamous cell carcinoma; HPV: human papillomavirus

In line with the technological progress, in the last few years several authors have focused their attention on the bacterial-induced EMT. For instance, Sztukowska et al. ${ }^{[38]}$ showed that $P$. gingivalis, associated with OPSCC development, is able in a gingival $3 \mathrm{D}$ equivalent to induce the expression and the nuclear relocalization of ZEB1, a well-known EMT transcriptional factor. This effect is reduced by the lack of the bacterial fimbrial FimA protein, while it is enhanced by the co-infection with F. nucleatum or S. gordonii. A similar study conducted by Lee et al ${ }^{[37]}$ showed that the carcinogenic properties of $P$. gingivalis are unrelated to the presence of chronic inflammation periodontitis. In this study, the authors showed that in addition to ZEB1, also glycogen synthase kinase-3 beta (p-GSK3 $\beta$ ), Slug, Snail, and vimentin usually increase during EMT under the influence of P. gingivalis. Moreover, the co-infection with F. nucleatum induces the loss of adhesive molecules such as E-cadherin and $\beta$-catenin in cancer-committed cells and the overexpression of MMP-2, -7 and -9 as showed for HPV infection ${ }^{[69]}$. These findings confirm those obtained in an established periodontitis-associated oral tumorigenesis murine model used to demonstrate that both $P$. gingivalis and F. nucleatum induce tumorigenesis independently from the inflammatory status ${ }^{[70]}$. In this latter study, the interaction of bacteria with the keratinocytes Toll-like receptor and the consequent activation of the IL-6-STAT3 axis was possibly involved in the tumorigenesis process.

The above cellular models can be also used to quantify the microorganisms' migration potential, as made by Dabija-Wolter et al ${ }^{[62]}$ who in vitro reconstructed a gingiva to evaluate the migration of $F$. nucleatum through a keratinized squamous epithelium without destroying it, while establishing a chronic infection [Figure 1].

Summarizing, in the last few years, thanks to their suitability to exceed the limitations of monolayer adherent cell cultures, as well as of in vivo models and spheroids, 3D epithelial co-cultures have become indispensable tools for investigating the interactions between microbial agents and their hosts. 
Although several questions are still open, soon further improvements might help to generate microenvironments that also mimic the bacteria and virus migratory potential.

\section{CONCLUSION}

Overall this review highlights the importance of the in vitro 3D modeling research field for the study of microbial induced epithelial carcinogenesis. Yet, further improvements are still needed to reach the level of complexity of the real tissue. These models will hopefully help to better understand the respective roles of virus and bacteria in HR-HPV-related cancers.

\section{DECLARATIONS}

\section{Acknowledgments}

Authors thank Michelle Schoeman for the linguistic revision.

\section{Authors' contributions}

Wrote part of the paper and prepared Table 1: Squarzanti DF

Wrote part of the paper and prepared Figure 1: Sorrentino R

Designed the review article and wrote part of the paper: Azzimonti B

\section{Availability of data and materials}

Not applicable.

\section{Financial support and sponsorship}

DFS was partially supported by BioLab Srl/Probiotical SpA.

\section{Conflicts of interest}

All authors declared that there are no conflicts of interest.

\section{Ethical approval and consent to participate}

Not applicable.

\section{Consent for publication}

Not applicable.

\section{Copyright}

(c) The Author(s) 2019.

\section{REFERENCES}

1. Human papillomavirus and related diseases report. HPV Information Center. Available from: https://hpvcentre.net/statistics/reports/XWX. pdf?t=1571975032537 [Last accessed on 25 Oct 2019]

2. Pouyanfard S, Müller M. Human papillomavirus first and second generation vaccines-current status and future directions. Biol Chem 2017;398:871-89.

3. Takes RP, Wierzbicka M, D'Souza G, Jackowska J, Silver CE, et al. HPV vaccination to prevent oropharyngeal carcinoma: what can be learned from anogenital vaccination programs? Oral Oncol 2015;51:1057-60.

4. Ferlay J, Ervik M, Lam F, Colombet M, Mery L, et al. Global Cancer Observatory: Cancer Today. Lyon, France: International Agency for Research on Cancer. 2018. Available from: https://gco.iarc.fr/today [Last accessed on 25 Oct 2019]

5. Squarzanti DF, Sorrentino R, Landini MM, Chiesa A, Pinato S, et al. Human papillomavirus type 16 E6 and E7 oncoproteins interact with the nuclear p53-binding protein 1 in an in vitro reconstructed 3D epithelium: new insights for the virus-induced DNA damage response. Virol J 2018; 15:176.

6. Deschler DG, Richmon JD, Khariwala SS, Ferris RL, Wang MB. The "new" head and neck cancer patient-young, nonsmoker, nondrinker, and HPV positive: evaluation. Otolaryngol Head Neck Surg 2014;151:375-80.

7. Sedghizadeh PP, Billington WD, Paxton D, Ebeed R, Mahabady S, et al. Is p16-positive oropharyngeal squamous cell carcinoma associated 
with favorable prognosis? A systematic review and meta-analysis. Oral Oncol 2016;54:15-27.

8. Nørregaard C, Grønhøj C, Jensen D, Friborg J, Andersen E, et al. Cause-specific mortality in HPV+ and HPV- oropharyngeal cancer patients: insights from a population-based cohort. Cancer Med 2018;7:87-94.

9. Sano D, Oridate N. The molecular mechanism of human papillomavirus-induced carcinogenesis in head and neck squamous cell carcinoma. Int J Clin Oncol 2016;21:819-26.

10. Kyrgiou M, Mitra A, Moscicki AB. Does the vaginal microbiota play a role in the development of cervical cancer? Transl Res 2017;179:168-82.

11. Belkaid Y, Harrison OJ. Homeostatic immunity and the microbiota. Immunity 2017;46:562-76.

12. Mitra A, MacIntyre DA, Marchesi JR, Lee YS, Bennett PR, et al. The vaginal microbiota, human papillomavirus infection and cervical intraepithelial neoplasia: what do we know and where are we going next? Microbiome 2016;4:58.

13. Mitra A, MacIntyre DA, Lee YS, Smith A, Marchesi JR, et al. Cervical intraepithelial neoplasia disease progression is associated with increased vaginal microbiome diversity. Sci Rep 2015;5:16865.

14. Perera M, Al-Hebshi NN, Speicher DJ, Perera I, Johnson NW. Emerging role of bacteria in oral carcinogenesis: a review with special reference to perio-pathogenic bacteria. J Oral Microbiol 2016;8:32762.

15. Al-Hebshi NN, Borgnakke WS, Johnson NW. The microbiome of oral squamous cell carcinomas: a functional perspective. Curr Oral Health Rep 2019;6:145-60.

16. Melissaridou S, Wiechec E, Magan M, Jain MV, Chung MK, et al. The effect of 2D and 3D cell cultures on treatment response, EMT profile and stem cell features in head and neck cancer. Cancer Cell Int 2019;19:16.

17. Ilhan ZE, Łaniewski P, Thomas N, Roe DJ, Chase DM, et al. Deciphering the complex interplay between microbiota, HPV, inflammation and cancer through cervicovaginal metabolic profiling. EBioMedicine 2019;44:675-90.

18. Rubinstein MR, Baik JE, Lagana SM, Han RP, Raab WJ, et al. Fusobacterium nucleatum promotes colorectal cancer by inducing Wnt/ $\beta$-catenin modulator Annexin A1. EMBO Rep 2019;20:e47638.

19. Mo Y, Wang Y, Zhang L, Yang L, Zhou M, et al. The role of Wnt signaling pathway in tumor metabolic reprogramming. J Cancer 2019;10:3789-97.

20. Olea-Flores M, Zuñiga-Eulogio MD, Mendoza-Catalán MA, Rodríguez-Ruiz HA, Castañeda-Saucedo E, et al. Extracellular-signal regulated kinase: a central molecule driving epithelial-mesenchymal transition in cancer. Int J Mol Sci 2019;20:e2885.

21. Timaner M, Tsai K, Shaked Y. The multifaceted role of mesenchymal stem cells in cancer. Semin Cancer Biol 2019;S1044-579X (19) 30135-X.

22. Cyprian FS, Al-Farsi HF, Vranic S, Akhtar S, Al Moustafa AE. Epstein-barr virus and human papillomaviruses interactions and their roles in the initiation of epithelial-mesenchymal transition and cancer progression. Front Oncol 2018;8:111.

23. Chen X, Bode AM, Dong Z, Cao Y. The epithelial-mesenchymal transition (EMT) is regulated by oncoviruses in cancer. FASEB J 2016;30:3001-10.

24. Lefevre M, Rousseau A, Rayon T, Dalstein V, Clavel C, et al. Epithelial to mesenchymal transition and HPV infection in squamous cell oropharyngeal carcinomas: the papillophar study. Br J Cancer 2017;116:362-9.

25. Jiang J, Li X, Yin X, Zhang J, Shi B. Association of low expression of E-cadherin and $\beta$-catenin with the progression of early stage human squamous cervical cancer. Oncol Lett 2019;17:5729-39.

26. Su PH, Hsu YW, Huang RL, Chen LY, Chao TK, et al. TET1 promotes 5hmC-dependent stemness, and inhibits a 5hmC-independent epithelial-mesenchymal transition, in cervical precancerous lesions. Cancer Lett 2019;450:53-62.

27. Li MY, Liu JQ, Chen DP, Li ZY, Qi B, et al. p68 prompts the epithelial-mesenchymal transition in cervical cancer cells by transcriptionally activating the TGF- $\beta 1$ signaling pathway. Oncol Lett 2018;15:2111-6.

28. Pang T, Li M, Zhang Y, Yong W, Kang H, et al. Y box-binding protein 1 promotes epithelial-mesenchymal transition, invasion, and metastasis of cervical cancer via enhancing the expressions of Snail. Int J Gynecol Cancer 2017;27:1753-60.

29. Kwon MJ, Rho YS, Nam ES, Cho SJ, Park HR, et al. Clinical implication of programmed cell death-1 ligand-1 expression in tonsillar squamous cell carcinoma in association with intratumoral heterogeneity, human papillomavirus, and epithelial-to-mesenchymal transition. Hum Pathol 2018;80:28-39.

30. Outh-Gauer S, Alt M, Le Tourneau C, Augustin J, Broudin C, et al. Immunotherapy in head and neck cancers: a new challenge for immunologists, pathologists and clinicians. Cancer Treat Rev 2018;65:54-64.

31. Zandberg DP, Strome SE. The role of the PD-L1: PD-1 pathway in squamous cell carcinoma of the head and neck. Oral Oncol 2014;50:62732.

32. Ma CT, Luo HS, Gao F, Tang QC, Chen W. Fusobacterium nucleatum promotes the progression of colorectal cancer by interacting with E-cadherin. Oncol Lett 2018;16:2606-12.

33. Olsen I, Yilmaz Ö. Possible role of Porphyromonas gingivalis in orodigestive cancers. J Oral Microbiol 2019;11:1563410.

34. Yan X, Liu L, Li H, Qin H, Sun Z. Clinical significance of Fusobacterium nucleatum, epithelial-mesenchymal transition, and cancer stem cell markers in stage III/IV colorectal cancer patients. Onco Targets Ther 2017;10:5031-46.

35. Panebianco C, Potenza A, Andriulli A, Pazienza V. Exploring the microbiota to better understand gastrointestinal cancers physiology. Clin Chem Lab Med 2018;56:1400-12.

36. Panebianco C, Andriulli A, Pazienza V. Pharmacomicrobiomics: exploiting the drug-microbiota interactions in anticancer therapies. Microbiome 2018;6:92.

37. Lee J, Roberts JS, Atanasova KR, Chowdhury N, Han K, et al. Human primary epithelial cells acquire an epithelial-mesenchymaltransition phenotype during long-term infection by the oral opportunistic pathogen, porphyromonas gingivalis. Front Cell Infect Microbiol 2017;7:493.

38. Sztukowska MN, Ojo A, Ahmed S, Carenbauer AL, Wang Q, et al. Porphyromonas gingivalis initiates a mesenchymal-like transition through ZEB1 in gingival epithelial cells. Cell Microbiol 2016;18:844-58. 
39. Abdulkareem AA, Shelton RM, Landini G, Cooper PR, Milward MR. Potential role of periodontal pathogens in compromising epithelial barrier function by inducing epithelial-mesenchymal transition. J Periodontal Res 2018;53:565-74.

40. Abdulkareem AA, Shelton RM, Landini G, Cooper PR, Milward MR. Periodontal pathogens promote epithelial-mesenchymal transition in oral squamous carcinoma cells in vitro. Cell Adh Migr 2018;12:127-37.

41. Sulaiman A, Yao ZM, Wang LS. Re-evaluating the role of epithelial-mesenchymal-transition in cancer progression. J Biomed Res 2018:32:81-90.

42. Yeung KT, Yang J. Epithelial-mesenchymal transition in tumor metastasis. Mol Oncol 2017;11:28-39.

43. Hofman P, Vouret-Craviari V. Microbes-induced EMT at the crossroad of inflammation and cancer. Gut Microbes 2012;3:176-85.

44. Gopinath D, Menon RK, Banerjee M, Su Yuxiong R, Botelho MG, et al. Culture-independent studies on bacterial dysbiosis in oral and oropharyngeal squamous cell carcinoma: A systematic review. Crit Rev Oncol Hematol 2019;139:31-40.

45. Todaro GJ, Zeve V, Aaronson SA. Cell culture techniques in the search for cancer viruses of man. In Vitro 1971;6:355-61.

46. Taylor-Robinson D. The use of organ cultures and animal models in the study of Mycoplasma pneumoniae infections. Infection 1976;4 (1 Suppl):4-8.

47. Bergmann S, Steinert M. From single cells to engineered and explanted tissues: new perspectives in bacterial infection biology. Inter Rev Cell Mol Biol 2015;319:1-44.

48. Pasupuleti MK, Molahally SS, Salwaji S. Ethical guidelines, animal profile, various animal models used in periodontal research with alternatives and future perspectives. J Indian Soc Periodontol 2016;20:360-8.

49. Riedl A, Schlederer M, Pudelko K, Stadler M, Walter S, et al. Comparison of cancer cells in 2D vs 3D culture reveals differences in AKTmTOR-S6K signaling and drug responses. J Cell Sci 2017;130:203-18.

50. Di Giulio M, Di Valerio V, Bosco D, Marsich E, Cataldi A, et al. Molecular mechanisms driving Streptococcus mitis entry into human gingival fibroblasts in presence of chitlac-nAg and saliva. J Mater Sci Mater Med 2018;29:36.

51. Millhouse E, Jose A, Sherry L, Lappin DF, Patel N, et al. Development of an in vitro periodontal biofilm model for assessing antimicrobial and host modulatory effects of bioactive molecules. BMC Oral Health 2014;14:80.

52. Spurgeon ME, Lambert PF. Human papillomavirus and the stroma: bidirectional crosstalk during the virus life cycle and carcinogenesis. Viruses 2017;9:E219.

53. Genovese NJ, Broker TR, Chow LT. Non conserved lysine residues attenuate the biological function of the low-risk human papillomavirus E7 protein. J Virol 2011;85:5546-54.

54. Banerjee NS, Wang HK, Broker TR, Chow LT. Human papillomavirus (HPV) E7 induces prolonged G2 following S phase reentry in differentiated human keratinocytes. J Biol Chem 2011;286:15473-82.

55. Chow MT, Luster AD. Chemokines in cancer. Cancer Immunol Res 2014;2:1125-31.

56. Bradbury P, Fabry B, O’Neill GM. Occupy tissue: the movement in cancer metastasis. Cell Adh Migr 2012;6:424-32.

57. Hogervorst M, Rietveld M, de Gruijl F, El Ghalbzouri A. A shift from papillary to reticular fibroblasts enables tumour-stroma interaction and invasion. Br J Cancer 2018;118:1089-97.

58. Fullar A, Dudas J, Olah L, Hollosi P, Papp Z, et al. Remodeling of extracellular matrix by normal and tumor-associated . broblasts promotes cervical cancer progression. BMC Cancer 2015;15:256.

59. de Carvalho Dias K, de Sousa DL, Barbugli PA, Cerri PS, Salih VM, et al. Development and characterization of a 3D oral mucosa model as a tool for host-pathogen interactions. J Microbiol Methods 2018;152:52-60.

60. Zhang M, Rose B, Lee CS, Hong AM. In vitro 3-dimensional tumor model for radiosensitivity of HPV positive OSCC cell lines. Cancer Biol Ther 2015;16:1231-40.

61. Sawant S, Dongre H, Singh AK, Joshi S, Costea DE, et al. Establishment of 3D co-culture models from different stages of human tongue tumorigenesis: utility in understanding neoplastic progression. PLoS Onep 2016;11:e160615.

62. Dabija-Wolter G, Sapkota D, Cimpan MR, Neppelberg E, Bakken V, et al. Limited in-depth invasion of Fusobacterium nucleatum into in vitro reconstructed human gingiva. Arch Oral Biol 2012;57:344-51.

63. Agarwal V, Kuchipudi A, Fulde M, Riesbeck K, Bergmann S, et al. Streptococcus pneumoniae endopeptidase O (PepO) is a multifunctional plasminogen and fi bronectin binding protein, facilitating evasion of innate immunity and invasion of host cells. J Biol Chem 2013;288,6849-63.

64. Agarwal V, Sroka M, Fulde, M, Bergmann S, Riesbeck K, et al. Binding of Streptococcus pneumoniae endopeptidase O (PepO) to complement component C1q modulates the complement attack and promotes host cell adherence. J Biol Chem 2014;289,15833-44.

65. Atala A, Kasper FK, Mikos AG. Engineering complex tissues. Sci Transl Med 2012;4:160rv12.

66. Rohde M, Chhatwal GS. Adherence and invasion of streptococci to eukaryotic cells and their role in disease pathogenesis. Curr Top Microbiol Immunol 2013;368:83-110.

67. McKenna DJ, Patel D, McCance DJ. MiR-24 and miR-205 expression is dependent on HPV onco-protein expression in keratinocytes. Virology 2014;448:210-6.

68. Bergmann S, Schoenen H, Hammerschmidt S. The interaction between bacterial enolase and plasminogen promotes adherence of Streptococcus pneumoniae to epithelial and endothelial cells. Int J Med Microbiol 2013;303,452-62.

69. Zhu D, Ye M, Zhang W. E6/E7 oncoproteins of high risk HPV-16 upregulate MT1-MMP, MMP-2 and MMP-9 and promote the migration of cervical cancer cells. Int J Clin Exp Pathol 2015;8,4981-9.

70. Gallimidi A, Fischman S, Revach B, Bulvik R, Maliutina A, et al. Periodontal pathogens Porphyromonas gingivalis and Fusobacterium nucleatum promote tumor progression in an oral-specific chemical carcinogenesis model. Oncotarget 2015;6:22613-23. 